\title{
Confidence interval in Kirsch equations
}

\author{
Morteza Khodabin ${ }^{1 *}$, Vahid Hosseinitoudeshki ${ }^{2}$, \\ ${ }^{1}$ Department of Mathematics, College of basic sciences, Karaj Branch, Islamic Azad University, Alborz, Iran \\ ${ }^{2}$ Department of Civil Engineering, Zanjan Branch, Islamic Azad University, Zanjan, Iran \\ * Corresponding Author: m-khodabin@kiau.ac.ir
}

\begin{abstract}
Copyright (C2014 Morteza Khodabin and Vahid Hosseinitoudeshki. This is an open access article distributed under the Creative Commons Attribution License, which permits unrestricted use, distribution, and reproduction in any medium, provided the original work is properly cited.
\end{abstract}

\begin{abstract}
Rocks at depth are affected by stresses resulting from the weight of the overlying strata and tectonic stresses. When a tunnel is excavated in this rock, the stress field is locally disordered and radial, tangential and shear stresses are induced in the rock around the tunnel. Knowledge of the magnitudes and directions of these induced stresses is essential. Since the measuring of specific gravity and depth are inevitably affected by environmental noise, we consider a random version of $P_{2}$ in Kirsch equations. By doing this, we define random version of the Kirsch equations. Then we introduce an algorithm to calculate confidence intervals for the Kirsch parameters. Finally we use Alborz tunnel characteristics for creating these confidence intervals as a case study. The results show that the proposed amounts of radial, tangential and shear stresses lie in desired range.
\end{abstract}

Keywords: Kirsch equations; Confidence interval; confidence level; Alborz tunnel; normal distribution.

\section{Introduction}

The maximum stress concentrations around tunnel openings are the tangential in nature and then could be calculated by using Kirsch equations (1898) in polar coordinate system. For calculation simplicity, the tunnel shapes were considered as circular. Furthermore, these equations are applicable for homogenous, isotropic, continuous and linearly elastic material. The Kirsch equations could be written as follows(Goodman, 1989):

$\sigma_{r}=\frac{P_{1}+P_{2}}{2}\left(1-\frac{a^{2}}{r^{2}}\right)+\frac{P_{1}-P_{2}}{2}\left(1-\frac{4 a^{2}}{r^{2}}+\frac{3 a^{4}}{r^{4}}\right) \cos 2 \theta$

$\sigma_{\theta}=\frac{P_{1}+P_{2}}{2}\left(1+\frac{a^{2}}{r^{2}}\right)-\frac{P_{1}-P_{2}}{2}\left(1+\frac{3 a^{4}}{r^{4}}\right) \cos 2 \theta$

$\tau_{r \theta}=-\frac{P_{1}-P_{2}}{2}\left(1+\frac{2 a^{2}}{r^{2}}-\frac{3 a^{4}}{r^{4}}\right) \sin 2 \theta$

In these expressions $p_{1}$ and $p_{2}$ are in-situ horizontal and vertical stresses respectively. $\sigma_{r}, \sigma_{\theta}$ and $\tau_{r \theta}$ are radial stress, tangential stress and shear stress in around tunnel, $a$ is radius of tunnel and $r$ is distance from center of tunnel. 


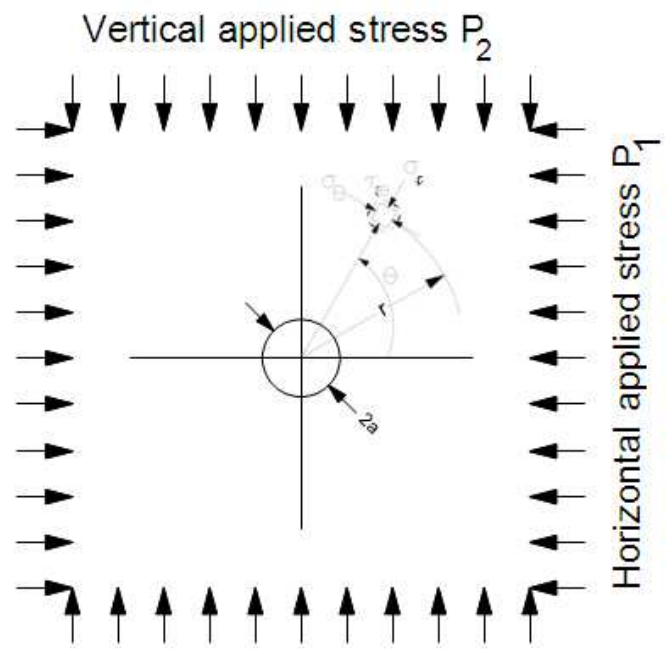

Figure 1: Stresses around a circular tunnel in an isotropic, linearly elastic, homogenous continuum

Assuming a principal stress direction is parallel to the tunnel axis and that the principal stress field in the plane perpendicular to the tunnel axis is anisotropic, the Kirsch equations and know of the rock material strength characteristics allow an assessment of the state of stress instantly outside the zone of influence of the tunnel (Brady and Brown, 2004). Since the Kirsch equations are based on in-situ stress values, therefore investigation of factors affecting them and confidence interval for stresses are necessary. In this paper a confidence interval are determined statistically for vertical stress values. The vertical stress in the rocks depends on weight of the overburden rocks and is calculated by multiplying density or specific gravity at depth of rocks. Density is a measure of mass per unit of volume. Density varies significantly among different rock types because of differences in mineralogy and porosity. Most rocks have density between $0.025 \mathrm{to} 0.028 \mathrm{MN} / \mathrm{m} 3$. Porosity describes how densely the material is packed. It is the ratio of the non-solid volume to the total volume of material. Porosity therefore is a fraction between 0 and 1 and varies depending on the amount of compression of rocks. It may also be represented in percent terms by multiplying the fraction by $100 \%$.

The density is sometimes defined by specific gravity. Specific gravity is the density of the rock relative to the density of water. Specific gravity is common physical properties. The most rocks have specific gravity between 2.5 to 2.8. In sedimentary rocks specific gravity is influenced by the compaction of rocks due to diagenesis and the amount of impurities and also the content of moisture. Specific gravity in igneous and metamorphic rocks is affected by composition of the minerals and presence or absence of pores. Therefore specific gravity can vary even in a certain rock depending on its compaction and impurities and so for calculating vertical stress to create a confidence interval for it values is necessary. This paper attempts to present the application of Kirsch equations for a circular tunnel with a $12 \mathrm{~m}$ diameter, excavated in Eocene Karaj tuffs and in depth of $300 \mathrm{~m}$. This tunnel is located in the Tehran-Shomal freeway and is to cross the Alborz Mountain Range in north of Iran. The specific gravity of tuffs is 2.55 and stresses around the tunnel are calculated in a distance of 9 meter and an angle $(\theta)$ of 30 degree.

Since the thrust faults are numerous and dominant in the Alborz Mountain Range (Alavi, 1994), the values of horizontal stresses are more than that of the vertical ones $\left(P_{1}>P_{2}\right)$ (Singh and Goel, 1999). These values were estimated for the this tunnel using Sengupta (1998, in Singh and Goel, 1999) equation, applied for steep mountain ranges like the Himalayan Region, which have the same tectonic settings and geological structures as the Alborz Range. For overburden less than $400 \mathrm{~m}$, Sengupta (1998, in Singh and Goel, 1999) has inferred that: $P_{1}=1.5+1.2 P_{2}$, where: $P_{2}=\gamma \times Z$. Accordingly, the vertical stress $\left(P_{2}\right)$ has been calculated as $7.65 \mathrm{MPa}$, considering the specific gravity values of 2.55 for the tuffs and the mean values of $300 \mathrm{~m}$ overburdens for the tunnel. Therefore, according to the Sengupta equation (1998, in Singh and Goel, 1999), the horizontal stress would be 10.62 MPa (Yassagi et al., 2005).

Then the stress concentrations around the tunnel are calculated by using Kirsch equation in polar coordinate system. Subsequently, the radial, tangential and shear stresses in around the tunnel and at distance of $9 \mathrm{~m}$ from center of tunnel at an angle of 30 degree are equal to 4.94, 12.007 and $1.67 \mathrm{MPa}$ respectively. 


\section{Random version of Kiresch equations}

Since the measuring of specific gravity and depth are inevitably affected by environmental noise, we must take the environmental noise into account in measuring the $p_{2}$. Let

$$
P_{2}^{\text {ran }}=P_{2}+" \text { Noise", }
$$

be random version of $P_{2}$, where "Noise" is the influence of environmental noise on specific gravity and depth, so, we can replace the "Noise" term by standard normal distribution random variable. i.e.

$$
P_{2}^{r a n}=P_{2}+\epsilon,
$$

where $\epsilon$ is a random variable with standard normal distribution. By this structure we get

$\sigma_{r r}=\frac{P_{1}+P_{2}^{r a n}}{2}\left(1-\frac{a^{2}}{r^{2}}\right)+\frac{P_{1}-P_{2}^{r a n}}{2}\left(1-\frac{4 a^{2}}{r^{2}}+\frac{3 a^{4}}{r^{4}}\right) \cos 2 \theta$

$\sigma_{\theta r}=\frac{P_{1}+P_{2}^{r a n}}{2}\left(1+\frac{a^{2}}{r^{2}}\right)-\frac{P_{1}-P_{2}^{r a n}}{2}\left(1+\frac{3 a^{4}}{r^{4}}\right) \cos 2 \theta$

$\tau_{r \theta r}=-\frac{P_{1}-P_{2}^{r a n}}{2}\left(1+\frac{2 a^{2}}{r^{2}}-\frac{3 a^{4}}{r^{4}}\right) \sin 2 \theta$

where, $\sigma_{r r}, \sigma_{\theta r}$ and $\tau_{r \theta r}$ are rendom version of $\sigma_{r}, \sigma_{\theta}$ and $\tau_{r \theta}$ respectively.

\section{Confidence intervals with $(1-\alpha) \%$ confidence level}

In this section, we use the following algorithm to construction of confidence intervals for Kirsch parameters.

A confidence interval gives an estimated range of values which is likely to include an unknown population parameter, the estimated range being calculated from a given set of sample data. If independent samples are taken repeatedly from the same population, and a confidence interval calculated for each sample, then a certain percentage(confidence level) of the intervals will include the unknown population parameter.

Confidence intervals are usually calculated so that this percentage is $95 \%$, but we can produce $90 \%, 99 \%$, $99.9 \%$ (or whatever) confidence intervals for the unknown parameter. The width of the confidence interval gives us some idea about how uncertain we are about the unknown parameter. A very wide interval may indicate that more data should be collected before anything very definite can be said about the parameter. Confidence intervals are more informative than the simple results of hypothesis tests (where we decide "reject $H_{0}$ " or "don't reject $H_{0}$ ") since they provide a range of plausible values for the unknown parameter.

Let $\epsilon \sim N\left(0, \sigma^{2}\right)$, then

$\sigma_{r r} \sim N\left(\sigma_{r}, \nu_{1}^{2} \sigma^{2}\right), \quad \sigma_{\theta r} \sim N\left(\sigma_{\theta}, \nu_{2}^{2} \sigma^{2}\right)$ and $\tau_{r \theta r} \sim N\left(\tau_{r \theta}, \nu_{3}^{2} \sigma^{2}\right)$

where,

$\nu_{1}=\frac{1}{2}\left(1-\frac{a^{2}}{r^{2}}\right)-\frac{1}{2}\left(1-\frac{4 a^{2}}{r^{2}}+\frac{3 a^{4}}{r^{4}}\right) \cos 2 \theta$.

$\nu_{2}=\frac{1}{2}\left(1+\frac{a^{2}}{r^{2}}\right)+\frac{1}{2}\left(1+\frac{3 a^{4}}{r^{4}}\right) \cos 2 \theta$.

$\nu_{3}=\frac{1}{2}\left(1+\frac{2 a^{2}}{r^{2}}-\frac{3 a^{4}}{r^{4}}\right) \sin 2 \theta$.

Suppose $X_{1}, \ldots, X_{n}$ is an independent sample from $\sigma_{r r}$ with (parameters) mean $\sigma_{r}$ and variance $\nu_{1}^{2} \sigma^{2}$. Let $\overline{\sigma_{r r}}=\left(X_{1}+\cdots+X_{n}\right) / n$ and $S^{2}=\frac{1}{n-1} \sum_{i=1}^{n}\left(X_{i}-\overline{\sigma_{r r}}\right)^{2}$, consequently the confidence interval for $\sigma_{r}$ is given by

$\overline{\sigma_{r r}}-t_{\frac{\alpha}{2}, n-1} \frac{\nu_{1} S}{\sqrt{n}} \leq \sigma_{r} \leq \overline{\sigma_{r r}}+t_{\frac{\alpha}{2}, n-1} \frac{\nu_{1} S}{\sqrt{n}}$. 
Similarly, suppose $X_{1}, \ldots, X_{n}$ is an independent sample from $\sigma_{\theta r}$ with mean $\sigma_{\theta}$ and variance $\nu_{2}^{2} \sigma^{2}$. Then the confidence interval for $\sigma_{\theta}$ is given by

$\overline{\sigma_{\theta r}}-t_{\frac{\alpha}{2}, n-1} \frac{\nu_{2} S}{\sqrt{n}} \leq \sigma_{\theta} \leq \overline{\sigma_{\theta r}}+t_{\frac{\alpha}{2}, n-1} \frac{\nu_{2} S}{\sqrt{n}}$.

Finally, suppose $X_{1}, \ldots, X_{n}$ is an independent sample from $\tau_{r \theta r}$ with mean $\tau_{r \theta}$ and variance $\nu_{3}^{2} \sigma^{2}$. Then the confidence interval for $\tau_{r \theta}$ is given by

$\overline{\tau_{r \theta r}}-t_{\frac{\alpha}{2}, n-1} \frac{\nu_{3} S}{\sqrt{n}} \leq \tau_{r \theta} \leq \overline{\tau_{r \theta r}}+t_{\frac{\alpha}{2}, n-1} \frac{\nu_{3} S}{\sqrt{n}}$.

\section{Case study}

Often the parameters required for stability analysis of tunnels are not well known. In the other words, stability analysis of tunnels is a geotechnical engineering problem characterized by many sources of uncertainty. In geomechanic problems, uncertainties get up from the difficulty in measuring key geomechanical characteristic such as stresses, modulus and strength of rocks. Rock at depth is subjected to stresses resulting from the weight of the overlying strata and from locked in stresses of tectonic origin. When a tunnel is excavated in this rock, the stress field is locally disrupted and a new set of stresses are induced in the rock surrounding the tunnel. Two factors that are related to the amount of these stresses and affecting the stability of tunnels are total displacements and radius of plastic zone around of tunnels. Displacements around of tunnels increases with increasing stresses and if the tangential stresses in tunnel surrounding rocks is more than half the ultimate strength of rocks, the plastic zone is formed. Therefore, accurate calculation of stresses around of tunnels is essential. Given the uncertainty in the calculated vertical stresses in the region due to specific gravity variations, determine a confidence interval for calculating the stresses is very usfull.

In this section, we use Alborz tunnel characteristics for creating above confidence intervals. This tunnel is a circular tunnel with a $6 \mathrm{~m}$ radius $(a)$ excavated in Eocene Karaj tuffs and in depth $(z)$ of $300 \mathrm{~m}$. The Alborz tunnel located in the Tehran-Shomal freeway and is to cross the Alborz Mountain Range in north of Iran. The specific gravity of tuffs is 2.55 , hence the unit weight of these rocks is $0.0255 \mathrm{MN} / \mathrm{m}^{3}$. Since the thrust faults are numerous and dominant in the Alborz Mountain Range (Alavi, 1994), the values of horizontal stresses are more than that of the vertical ones $\left(P_{1}>P_{2}\right)$ (Singh and Goel, 1999). These values were estimated for the this tunnel using Sengupta (1998, in Singh and Goel, 1999) equation, applied for steep mountain ranges like the Himalayan Region, which have the same tectonic settings and geological structures as the Alborz Range. For overburden less than $400 \mathrm{~m}$, Sengupta (1998, in Singh and Goel, 1999) has inferred that: $P_{1}=1.5+1.2 P_{2}$, where: $P_{2}=\gamma \times Z$ ( $\gamma$ is unit weight). Accordingly, the vertical stress $\left(P_{2}\right)$ has been calculated as $7.65 \mathrm{MPa}$, considering the specific gravity values of 2.55 for the tuffs and the mean values of $300 \mathrm{~m}$ overburdens for the tunnel. Therefore, according to the Sengupta equation (1998, in Singh and Goel, 1999), the horizontal stress would be $10.62 \mathrm{MPa}$ (Yassagi et al., 2005). Then the stress concentrations around the tunnel are calculated by using Kirsch equation in polar coordinate system. Subsequently, the radial, tangential and shear stresses in around the tunnel and at distance of $9 \mathrm{~m}$ from center of tunnel at an angle of 30 degree are equal to 4.94, 12.007 and $1.67 \mathrm{MPa}$ respectively.

For do this, we use the following algorithm.

\section{Algorithm:}

1- We may assume, without any loss of generality, that $\sigma^{2}=1$.

2- Calculate one value for each of these parameters as deterministic case.

3- Select a sample from your chosen population.Generate 10000 random data from normal distribution with corresponding mean and variance (7 relation).

4- Choose your desired confidence level. The most commonly used confidence levels are 90 percent, 95 percent and 99 percent. This may also be provided for you in the course of a problem. Let's say you've chosen $95 \%$.

5- Calculate your margin of error $\left(t_{\frac{\alpha}{2}, n-1} \frac{\nu_{i} S}{\sqrt{n}}\right)$.

6-Add and subtract sample mean to margin of error for finding upper and lower bound.

7- State your confidence interval.

The following table summarizes the Results. 
Table 1: Confidence Interval for Kirsch parameters

\begin{tabular}{cccccc}
\hline Variable & $\mathrm{n}$ & Mean & StDev & SE Mean & $95 \%$ CI \\
\hline$\sigma_{r}$ & 10000 & 4.93999 & 0.00323 & 0.00003 & $(4.93992 ; 4.94005)$ \\
$\sigma_{\theta}$ & 10000 & 12.0070 & 0.0113 & 0.0001 & $(12.0068 ; 12.0072)$ \\
$\tau_{\theta r}$ & 10000 & -1.66997 & 0.00566 & 0.00006 & $(-1.67008 ;-1.66986)$ \\
\hline
\end{tabular}

\section{Conclusion}

In this study, the proposed amounts of radial, tangential and shear stresses lie in desired range. Therefore, this analysis covers the defects of a non-random sampling. As result, the current researchers recommend that future researches use applied algorithm in this paper for calculating stresses around tunnels.

\section{References}

[1] Alavi M. Tectonostratigraphic synthesis and structural style of the Alborz mountain system in northern Iran J Geodyn 1994; 21: 1-33.

[2] Brady BHG, Brown ET. Rock Mechanics for Underground Mining, 1st edn Allen and Unwin, London 2004.

[3] Casella G, and Berger R. Statistical Inference, 2rd ed, R. R. Donnelley and Sons Co, Craw-fordsville 2001.

[4] Goodman RE. Introduction to Rock Mechanics. Wiley, New York 1989; 478 pp.

[5] Jaeger C. Rock Mechanics and Engineering, Cambridge University Press 1979; 523 pp.

[6] Kirsch G. Die theorie der elastizitat und die bedurfnisse der festigkeitslehre. Veit Ver Deut Ing 1898; $42: 797-807$.

[7] Singh B, Goel RK. Rock Mass Classification, A Practical Approach in Civil Engineering. Elsevier, Amsterdam 1999; 265 pp.

[8] Yassaghi A, Salari-Rad H, Kanani-Moghadam H. Geomechanical evaluations of Karaj tuffs for rock tunneling in TehranShomal Freeway, Iran Engineering Geology 2005; 77: 83-98. 